\title{
Análise enantiosseletiva de fármacos e metabólitos empregando eletroforese capilar
}

\author{
Anderson Rodrigo Moraes de Oliveira \\ Departamento de Química, Faculdade de Filosofia, Ciências e Letras de Ribeirão Preto, \\ Universidade de São Paulo, Cep 14040-901, Ribeirão Preto, SP, Brasil \\ e-mail:deoliveira@usp.br
}

\section{Resumo}

Este artigo tem como objetivo discutir os fundamentos da análise enantiosseletiva de fármacos e metabólitos, empregando a eletroforese capilar como técnica de análise. Aspectos teóricos envolvendo os principais seletores quirais empregados nas análises são apresentados, assim como aplicações em diversas áreas.

\section{Palavras-chave}

Eletroforese capilar; análise enantiosseletiva; fármacos; metabólitos.

\section{Enantioselective analysis of drugs and metabolites by capillary electrophoresis}

\section{Abstract}

This article aims to discuss the basics of enantioselective analysis of drugs and metabolites using capillary electrophoresis as analytical technique. The main theoretical aspects involving chiral selectors used in the analysis are presented as well as applications in various areas.

Keywords

Capillary electrophoresis; enantioselective analysis; drugs; metabolites.

\section{Introdução}

A história da estereoquímica começou há mais de 150 anos atrás, quando Louis Pasteur demonstrou que quando um feixe de luz polarizada incidia através de soluções de ácido tartárico, o feixe de luz planopolarizada girava diferentemente. Pasteur também observou que este ácido, quando cristalizado, formava cristais em duas diferentes formas que eram imagens especulares uma da outra. As espécies que são imagens especulares não sobreponíveis são chamadas de enantiômeros. Os enantiômeros diferem um do outro apenas no arranjo espacial dos 
ligantes ao redor do centro quiral. Os enantiômeros possuem as mesmas propriedades físico-químicas (por exemplo, ponto de fusão, solubilidade) em um ambiente aquiral. No entanto, em um ambiente quiral, os enantiômeros podem se comportar diferentemente. Uma mistura de dois enantiômeros na mesma proporção é chamada de racemato $^{[1]}$. Na natureza, muitos dos processos essenciais para a vida são estereoespecíficos e centros quirais são comuns em biomoléculas. Alvos farmacológicos, tais como receptores, enzimas e canais iônicos, são, muitas vezes, moléculas quirais. Portanto, quando fármacos quirais são introduzidos no organismo, poderá haver um grau de discriminação quiral com a atividade farmacológica dos enantiômeros podendo ser marcadamente diferente, como consequência da interação com esses ambientes biológicos assimétricos. Essas interações distintas podem resultar em diferenças nos parâmetros farmacocinéticos, eficácia ou toxicidade dos medicamentos $^{[2]}$.

Compreender a contribuição da estereoquímica na ação e disposição de fármacos é fundamental para o êxito do desenvolvimento de medicamentos quirais ${ }^{[3]}$, bem como para determinar se o fármaco já desenvolvido ou utilizado como mistura racêmica pode ser substituído pelo mais seguro ou eficaz enantiômero puro desse fármaco ${ }^{[4]}$. No entanto, essa decisão exige uma justificativa científica baseada na qualidade, segurança e eficácia do fármaco ${ }^{[5]}$. Como resultado, vários novos medicamentos vêm sendo comercializados como enantiômeros puros. Contudo, há também exemplos de novos fármacos que eram ou são comercializados como misturas racêmicas e hoje são comercializados como enantiômeros puros ${ }^{[6]}$.
Assim, a procura de métodos analíticos adequados para determinação precisa, exata e reprodutível dos enantiômeros em diversas amostras tem aumentado significativamente nos últimos $\operatorname{anos}^{[7]}$.

Várias técnicas podem ser empregadas na análise enantiosseletiva de fármacos, tais como cromatografia líquida de alta eficiência (HPLC), cromatografia gasosa (GC) e eletroforese capilar (CE). Dentre essas técnicas, a HPLC é a técnica mais empregada em análise enantiosseletiva. Contudo, o uso da eletroforese capilar vem aumentando significativamente devido às inúmeras vantagens dessa técnica, tais como: baixo custo de análise, ausência ou reduzido consumo de solventes orgânicos, alta seletividade e excelente eficiência na separação dos enantiômeros. Este artigo tem como objetivo apresentar a CE como técnica na análise enantiosseletiva de fármacos e metabólitos, enfatizando os princípios teóricos da técnica, assim como demonstrar alguns exemplos de aplicações práticas em diversas áreas.

\section{Análise enantiosseletiva empregando a eletroforese capilar}

A separação de enantiômeros por CE pode ser feita de duas maneiras, empregando o modo indireto ou modo direto de separação ${ }^{[7]}$. No modo indireto a separação é baseada na formação de diastereoisômeros estáveis após a reação com um agente de derivatização quiral. Os resultantes diastereoisômeros podem então serem separados, com base em suas diferentes propriedades físico-químicas, usando uma solução tampão convencional. 
No modo direto de separação, seletores quirais são adicionados na solução tampão de análise e complexos diastereoisoméricos transitórios são formados. Tais complexos poderão ter mobilidades diferentes, atingindo o detector em tempos diferentes. Atualmente, a grande maioria das separações enantioméricas por CE é realizada empregando o modo direto, com adição de seletores quirais na solução tampão de análise. Portanto, este artigo dará enfase a essa modalidade de separação.

$\mathrm{Na}$ análise enantiosseletiva por $\mathrm{CE}$ com o uso de seletores quirais adicionados na solução tampão de análise, dois mecanismos de separação podem ser considerados, um cromatográfico e outro eletroforético ${ }^{[8,9]}$. O reconhecimento estereosseletivo dos enantiômeros pelos seletores quirais é um princípio cromatográfico. Normalmente os seletores quirais estão dissolvidos na solução tampão de análise, formando assim, uma pseudofase estacionária. Por outro lado, o princípio eletroforético da técnica também influencia a migração do analito, do seletor quiral e do complexo analito-seletor quiral em direção ao detector. Dessa forma, alguns autores chamam essa técnica de eletrocromatografia capilar $^{[8,9]}$ e não somente eletroforese capilar.

A resolução dos enantiômeros é baseada em um equilíbrio de complexação e pode ser descrito de acordo com a Equação $1^{[8,10]}$ :

$$
\Delta \mu=\mu_{R}-\mu_{S} \frac{\mu_{j}+\mu_{c R} K_{R}[C]}{1+K_{R}[C]}-\frac{\mu_{j}+\mu_{c S} K_{S}[C]}{1+K_{S}[C]}
$$

Onde $\mu_{R}$ e $\mu_{S}$ são as mobilidades observadas dos enantiômeros R- e S-, respectivamente; $\mu_{f}$ é a mobilidade dos enantiômeros não complexados; $\mathrm{K}_{\mathrm{R}}$ e $\mathrm{K}_{\mathrm{S}}$ são as constantes de equilíbrio de complexação dos enantiômeros R- e S- com o seletor quiral, respectivamente; $\mu_{c R}$ e $\mu_{c S}$ são as mobilidades dos respectivos complexos diastereoisoméricos temporários entre o seletor quiral e os enantiômeros R- e S-; e [C] é a concentração do seletor quiral. Enquanto as mobilidades dos enantiômeros livres são iguais, as constantes de equilíbrio e/ou a mobilidade dos complexos diastereoisoméricos formados podem ser diferentes. Considerando uma separação baseada nas diferenças das constantes de complexação, as mobilidades dos analitos livres e complexados devem ser diferentes para se conseguir a separação dos enantiômeros. Derivando a Equação 1, uma enantiosseparação pode ser obtida no caso de constantes diferentes $\left(\mathrm{K}_{\mathrm{R}} \neq \mathrm{K}_{\mathrm{S}}\right)$ ou, alternativamente, no caso de mobilidades diferentes dos complexos formados $\left(\mu_{\mathrm{cR}} \neq \mu_{\mathrm{cS}}\right)$. No primeiro caso, a enantiosseparação é somente devido à diferença nas constantes de complexação, de acordo com a Equação 2, abaixo ${ }^{[8,10]}$ :

$$
\Delta \mu=\frac{\left(\mu_{j}-\mu_{c}\right)\left(K_{R}-K_{S}\right)[C]}{1+\left(K_{R}+K_{S}\right)[C]+K_{R} K_{S}[C]^{2}}
$$

No caso de mobilidades diferentes do complexo, a Equação 1 pode ser ainda mais simplificada, de acordo com a Equação 3, abaixo ${ }^{[8,10]}$ :

$$
\Delta \mu=\frac{K[C]\left(\mu_{c R}-\mu_{c S}\right)}{1+K[C]}
$$

Dessa forma, a separação enantiosseletiva dos enantiômeros pode ser alcançada devido a diferentes constantes de ligação $\left(K_{R} \neq K_{S}\right)$ ou devido a diferentes mobilidades do complexo formado $\left(\mu_{c R} \neq \mu_{c S}\right)$.

\section{Seletores quirais empregados em CE}

Diversos seletores quirais podem ser utilizados em eletroforese capilar para separação de enantiômeros. Uma característica fundamental dos seletores quirais é apresentar estabilidade química e estabilidade quiral ${ }^{[11]}$. Essas estabilidades são fundamentais tanto no estado sólido 
como na solução tampão de análise. A falta de estabilidade quiral é observada quando ocorre a racemização do seletor quiral. Além disso, é fundamental que o seletor quiral seja solúvel na solução tampão de análise. Entre esses se destacam as: (i) ciclodextrinas e seus derivados, (ii) seletores baseados em surfactantes quirais, (iii) seletores quirais baseados em antibióticos macrocíclicos, (iv) seletores quirais baseados em éteres de coroa, (iv) seletores quirais baseados em proteínas e (v) misturas de dois ou mais seletores quirais, conhecidos como sistemas dual system. Além desses seletores, existem ainda os seletores quirais por troca de ligantes, seletores quirais baseados em polissacarídeos e reagentes para formação de par iônico quiral. Sem dúvida alguma, o seletor que mais se destaca entre esses são as ciclodextrinas e seus derivados. Neste artigo, serão discutidos os seletores enumerados de i-v. Para maiores detalhes sobre outros seletores quirais, os leitores podem consultar as referências bibliográficas no final deste artigo.

\subsection{Ciclodextrinas e seus derivados}

Ciclodextrinas (CDs) são os seletores quirais mais empregados em análise enantiosseletiva por eletroforese capilar. Essas moléculas possuem diversas propriedades que tornam seu uso atraente tais como: baixa absorção no UV, possibilidade de obtenção de CDs com diferentes polaridades e solubilidades e alto grau de enantiodiscriminação. As CDs são oligossacarídeos quirais cíclicos obtidas pela degradação enzimática do amido. Essas moléculas têm o formato de um cone truncado com uma cavidade interna, relativamente hidrofóbica e, devido à presença de grupos hidroxila nas bordas da cavidade, essas moléculas possuem também uma região hidrofílica $^{[12]}$. As ciclodextrinas nativas, que ocorrem naturalmente, são: $\alpha-, \beta-, \gamma$-CDs, compostas por seis, sete e oito unidades de anidroglicopiranose, respectivamente $^{[13]}$ (Figura 1).

O mecanismo mais aceito para separação dos enantiômeros com as CDs envolve a inclusão do analito quiral em sua cavidade e o estabeleci-

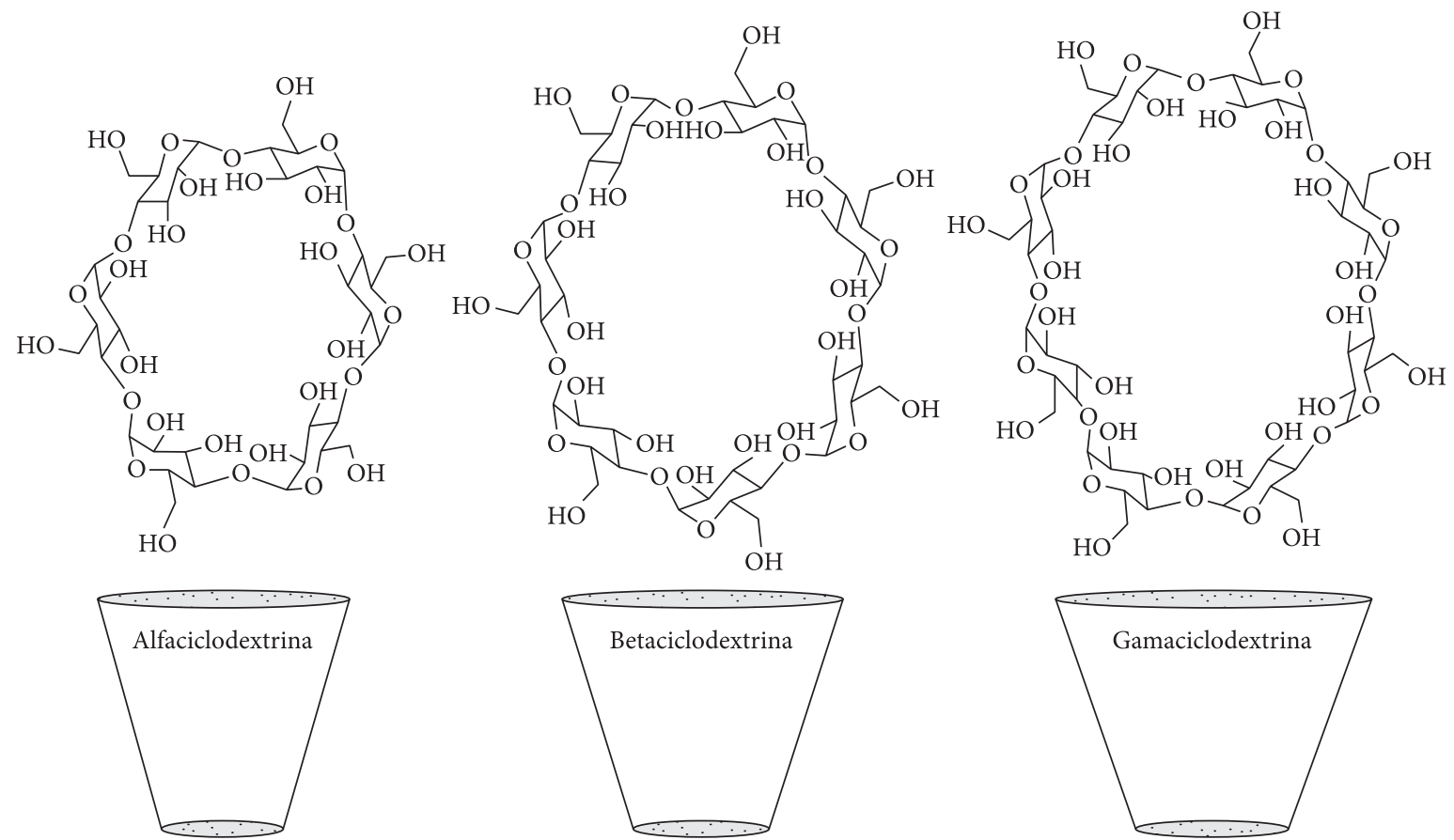

Figura 1 Estrutura química e esquema da $\alpha$ - $\beta$ - e $\gamma$ - ciclodextrinas. 
mento de interações secundárias com os grupos hidroxila nas bordas da cavidade da CD. A diferença nas constantes de inclusão entre os enantiômeros e as CDs é responsável pela enantiorresolução, pois os diasteroisômeros transitórios formados terão tempos de migração diferentes ${ }^{[13]}$.

Algumas CDs nativas apresentam um baixo poder de separação dos enantiômeros, portanto diversos derivados vêm sendo sintetizados empregando essas CDs como "molde". As CDs derivadas são moléculas com um alto poder de resolução e com diferentes características químicas. Dessa forma, quatro grupos diferentes de derivados podem ser distinguidos: as CDs i) neutras, ii) aniônicas, iii) catiônicas e iv) anfóteras.

\subsubsection{Ciclodextrinas neutras}

As CDs neutras são obtidas pela substituição de grupos hidroxila por grupos alquila ou hidroxialquila, o que aumenta a solubilidade e flexibilidade das $\mathrm{CDs}^{[14]}$. Alguns exemplos desta classe de CD são: 2-hidroxipropil- $\beta$-CD, dimetil- $\beta-C D$, trimetil- $\beta-\mathrm{CD}$, hidroxietil-CD, heptakis-(2,6-diO-metil)- $\beta$-CD, heptakis-(2,3, 6-tri-O-metil)$\beta$-CD. As heptakis são CDs em suas formas enantioméricas puras tendo, portanto, um custo mais elevado. As CDs neutras são utilizadas na separação de analitos ionizados ${ }^{[13]}$ que apresentam algum grau de mobilidade eletroforética.
Assim, a separação de bases ionizáveis é frequentemente realizada em meio ácido, enquanto que a separação de ácidos ionizáveis é realizada empregando um meio mais alcalino. A Tabela 1 apresenta alguns exemplos de separações quirais, empregando CDs neutras. A Figura 2 apresenta a separação quiral da terbutalina, um medicamento beta-agonista adrenérgico empregado no tratamento de doenças respiratórias.

\subsubsection{Ciclodextrinas aniônicas}

As CDs neutras possuem limitada enantiorresolução e analitos neutros não podem ser separados. Assim, CDs carregadas vêm sendo desenvolvidas. Essas ciclodextrinas podem separar analitos não ionizados ou ionizados. Portanto, a mobilidade eletroforética da CD e do analito são importantes na separação quiral com CDs ionizadas.

As CDs aniônicas mais empregadas são: carboximetil- $\beta-C D(C M-\beta-C D)$, carboxietil- $\beta-C D$, succinil- $\beta-C D$, sulfobutiléter- $\beta-C D(S B-\beta-C D)$ e sulfoetil- $\beta-C D$ e $C D-\beta$-sulfatada (S- $\beta-C D)$. CDs aniônicas, como a S- $\beta-C D$ e SB- $\beta-C D$, são carregadas negativamente em qualquer faixa de $\mathrm{pH}$ e são usadas principalmente para a separação de compostos quirais neutros e básicos ${ }^{[21,22]}$. Por outro lado, a CM- $\beta$-CD contém grupos carboxílicos e sua carga depende do pH da solução tampão de análise. Em

Tabela 1 Alguns exemplos de separações quirais empregando CDs neutras.

\begin{tabular}{lcccc}
\hline \multicolumn{1}{c}{ Analito } & Seletor quiral & $\mathrm{pH}$ da solução tampão & Matriz & Ref. \\
\hline Amlodipina & HP- $\beta-C D$ & 2,5 & Soro & 15 \\
Cetoprofeno & TM- $\beta-C D$ & 5,0 & Soro & 16 \\
Metotrexato & $\beta-C D$ & 9,3 & Formulação farmacêutica & 17 \\
Etodolaco & HP- $\beta-C D$ & 7,0 & Formulação farmacêutica & 18 \\
Baclofeno & $\alpha-C D$ & 9,5 & Plasma & 19 \\
Clenbuterol & DM- $\beta-C D$ & 2,5 & Plasma & 20 \\
\hline
\end{tabular}

HP- $\beta-C D$, hidroxipropil-beta-ciclodextrina; TM- $\beta-C D$, trimetil-beta-ciclodextrina; $\beta-C D$, beta-ciclodextrina, $\alpha-C D$, alfa-ciclodextrina; DM- $\beta-C D$, dimetil-beta-ciclodextrina. 
pH baixo (2,5), essa CD não está ionizada e se comporta como uma CD neutra. Em pH acima de 5, essa $\mathrm{CD}$ está completamente ionizada. Assim, o pH do meio deve ser avaliado quando a CM- $\beta$-CD for empregada. Ciclodextrinas fosfatadas também vêm sendo desenvolvidas, porém seu uso ainda é muito

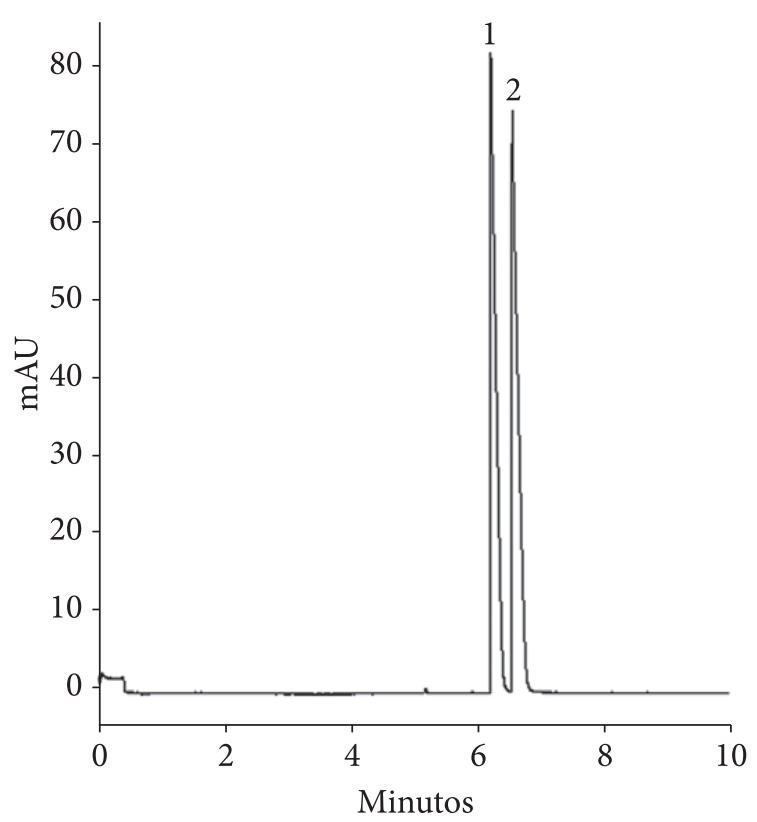

Figura 2 Eletroferograma referente à análise dos enantiômeros da terbutalina. Tampão de análise: tampão fosfato $50 \mathrm{mmol} . \mathrm{L}^{-1} \mathrm{pH} \mathrm{2,5}$ contendo HP- $\beta$ CD $15 \mathrm{mmol} . \mathrm{L}^{-1}$. Temperatura de análise de $20^{\circ} \mathrm{C}$, tensão aplicada de $+30 \mathrm{kV}$. (1) Enantiômero 1; (2) Enantiômero 2, detecção em 205 nm. limitado $^{[23]}$. Assim, como nas CDs neutras, CDs aniônicas formadas por isômeros isolados também vêm sendo sintetizadas, podendo-se citar: hexakis (2,3-diacetil-6-O-sulfo)- $\alpha$-CD, hexakis (2,3-di-Ometil-6-O-sulfo)- $\alpha-\mathrm{CD}$, hexakis (6-O-sulfo)- $\alpha-\mathrm{CD}$, heptakis (2,3-diacetil-6-O-sulfo)- $\beta$-CD, heptakis (2,3-di-O-metil-6-O-sulfo)- $\beta$-CD ${ }^{[11,24]}$. A Tabela 2 apresenta algumas aplicações das CDs aniônicas na análise enantiosseletiva de compostos quirais. A Figura 3 apresenta a separação quiral do ricobendazol (também conhecido como albendazol sulfóxido), um medicamento anti-helmíntico, utilizando a betaciclodextrina sulfobutiléter.

\subsubsection{Ciclodextrinas catiônicas}

As CDs catiônicas são usadas para a separação de compostos quirais ácidos, básicos e neutros ${ }^{[33,34]}$, mas não têm sido empregadas com muita frequência na separação de fármacos e metabólitos. A CD catiônica mais usada é a 2-hidroxipropil-3-trimetilamônio- $\beta$-CD (QA- $\beta-C D)$. Sua principal aplicação é na separação de ácidos carboxílicos e aminoácidos. QA- $\beta$-CD contém grupos de amônio quaternário, que são carregados em qualquer faixa de $\mathrm{pH}^{[21]}$. Essa $\mathrm{CD}$ apresenta uma mobilidade eletroforética independente do $\mathrm{pH}$ da solu-

Tabela 2 Alguns exemplos de separações quirais empregando CDs aniônicas.

\begin{tabular}{|c|c|c|c|c|}
\hline Analito & Seletor quiral & $\begin{array}{l}\text { pH da solução } \\
\text { tampão }\end{array}$ & Matriz & Ref. \\
\hline Amissulprida & $S-\beta-C D$ & 3,5 & Formulação farmacêutica & 25 \\
\hline Ibuprofeno & $S-\beta-C D$ & 2,6 & Plasma & 26 \\
\hline Efedrina e pseudoefedrina & $S-\gamma-C D$ & 2,6 & Metanfetamina apreendida & 27 \\
\hline Mirtazapina e metabólitos & $\mathrm{CM}-\beta-\mathrm{CD}$ & 2,5 & Plasma & 28 \\
\hline Mirtazapina e metabólitos & $\mathrm{CM}-\beta-\mathrm{CD}$ & 2,5 & Urina & 29 \\
\hline Ofloxacina e metabólitos & $S B-\beta-C D$ & 2,0 & Urina & 30 \\
\hline Feniramina e metabólitos & $C E-\beta-C D$ & 4,5 & Urina & 31 \\
\hline Cetirizina & $S-\beta-C D$ & 8,7 & Plasma & 32 \\
\hline
\end{tabular}

S- $\beta$-CD, ciclodextrina-beta-sulfatada; S- $\gamma-C D$, ciclodextrina-gama-sulfatada; CM- $\beta-C D$, carboximetil-betaciclodextrina; CE- $\beta-C D$, carboxietil-beta-ciclodextrina; SB- $\beta-C D$, sulfobutiléter-beta-ciclodextrina. 


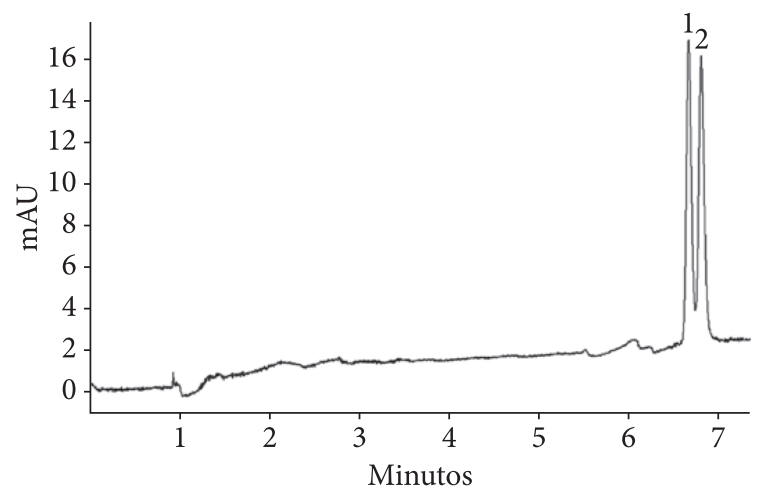

Figura 3 Eletroferograma referente à análise dos enantiômeros do ricobendazol. Tampão de análise: tris(hidroximetil)aminometano 25 mmol.L-1 $\mathrm{pH}$ 9,3, contendo SBE- $\beta-C D 4 \%+10 \%$ de acetonitrila. Temperatura de análise de $25{ }^{\circ} \mathrm{C}$, tensão aplicada de +25 kV. (1) Enantiômero 1; (2) Enantiômero 2, detecção em 220 nm.

ção tampão. Além disso, o fluxo eletrosmótico da solução é invertido quando compostos de amônio quaternário são utilizados ${ }^{[11]}$. Somente pequenas quantias dessa $\mathrm{CD}$ são necessárias para a separação quiral. Liu et al. ${ }^{[35]}$ desenvolveram a separação enantiomérica de um dissulfato orgânico altamente carregado negativamente. Este composto é um análogo estilbeno dissulfonato que possui interessantes propriedades em membranas. A separação dos enantiômeros foi realizada com uma solução tampão glicina com 5 mmol.L $L^{-1}$ de QA- $\beta$-CD. $\mathrm{O}$ método foi utilizado para avaliar a pureza enantiomérica após a síntese estereosseletiva do composto.

\subsubsection{Ciclodextrinas anfóteras}

Dependendo do $\mathrm{pH}$ da solução tampão de análise, estas CDs podem se comportar como aniônicas, neutras ou catiônicas ${ }^{[36]}$. Um exemplo de CD anfótera é o cloreto de 2-hidroxipropiltrimetilamônio. Existem poucas aplicações com estas CDs. Alguns racematos ácidos (ácido 4-bromomandélico, ácido 4-cloromandélico, ácido crisântemo monocarboxílico, ácido 3-fenilbutí- rico e ácido 2-fenoxipropiónico) foram separados usando uma CD- $\beta$ anfotérica (AM- $\beta-C D$ ) como seletor quiral ${ }^{[37]}$. Neste estudo foi empregado um capilar recoberto com poliacrilamida, polaridade reversa de $-12 \mathrm{kV}$ e solução tampão fosfato $50 \mathrm{mmol} \cdot \mathrm{L}^{-1}$. A concentração do seletor quiral foi de $20 \mathrm{mmol} . \mathrm{L}^{-1}$. Neste estudo foi possível obter a resolução de linha de base de todos os analitos avaliados.

\subsection{Seletores quirais baseados em surfactantes quirais}

Surfactantes são moléculas anfifílicas constituídas por um grupamento polar (cabeça polar) e um grupamento apolar (cauda apolar). Quando empregados acima de sua concentração micelar crítica (CMC), ocorre a formação de micelas. Como exemplo, o surfactante SDS ou dodecil sulfato de sódio acima de $8,1 \mathrm{mmol} . \mathrm{L}^{-1}$ encontra-se sob a forma micelar.

Portanto, micelas são agregados cuja superfície é formada por uma cabeça polar e o interior é apolar, devido à cauda hidrofóbica do surfactante (em solução aquosa). Se essas micelas forem formadas por moléculas quirais, então ocorrerá a formação de uma pseudofase estacionária quiral no interior do capilar. $\mathrm{O}$ uso de surfactantes quirais foi introduzido por Terabe et al. ${ }^{[38]}$. Devido à formação dessa pseudofase estacionária, essa técnica foi chamada de cromatografia eletrocinética micelar (MECK). Diferentes tipos de surfactantes quirais (aniônicos, catiônicos, neutros e surfactantes anfóteros) podem ser empregados, tais como: sais biliares, saponinas, $n$-alquil-L-aminoácidos de cadeia longa, $n$-alcanoil-L-aminoácidos, alquilglicosídeos e outros $^{[39,40]}$. A Figura 4 ilustra um esquema geral de um sal biliar e seus possíveis derivados. 
O mecanismo de separação quiral que envolve esses seletores é baseado nas interações estereoespecíficas entre o enantiômero e as micelas quirais. A discriminação quiral em MECK envolve a formação de complexos diastereoisoméricos temporários entre os enantiômeros do analito e o surfactante quiral. Os enantiômeros são separados quando suas constantes de ligação com o surfactante e/ou as mobilidades dos complexos diastereoisoméricos formados são diferentes ${ }^{[41]}$. Contudo, deve ser ressaltado que as interações diastereoisoméricas são depen-

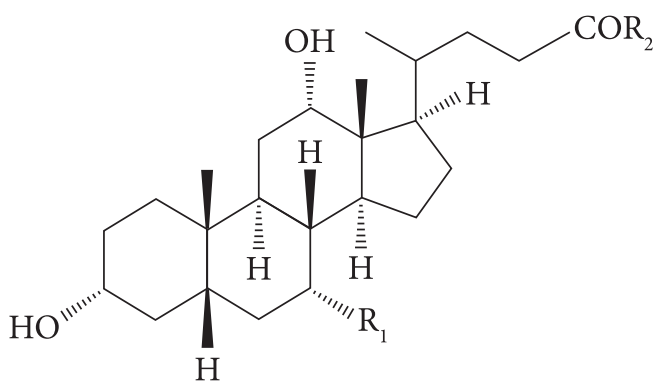

$$
\begin{array}{cl}
\text { Colato de sódio } & \multicolumn{1}{c}{\text { Taurocolato de sódio }} \\
\mathrm{R}_{1}=\mathrm{OH} & \mathrm{R}_{1}=\mathrm{OH} \\
\mathrm{R}_{2}=\mathrm{ONa} & \mathrm{R}_{2}=\mathrm{NHCH}_{2} \mathrm{CH}_{2} \mathrm{SO}_{3} \mathrm{Na}
\end{array}
$$

Deoxicolato de sódio Taurodeoxicolato de sódio

$$
\begin{array}{ll}
\mathrm{R}_{1}=\mathrm{H} & \mathrm{R}_{1}=\mathrm{H} \\
\mathrm{R}_{2}=\mathrm{ONa} & \mathrm{R}_{2}=\mathrm{NHCH}_{2} \mathrm{CH}_{2} \mathrm{SO}_{3} \mathrm{Na}
\end{array}
$$

Figura 4 Esquema geral de um seletor quiral baseado em um sal biliar e seus possíveis derivados. dentes do coeficiente de partição do analito entre as micelas e a fase aquosa total, em que as micelas estão dissolvidas. Na separação quiral por MECK, o centro quiral do surfactante está localizado próximo ao grupamento polar, que constitui a camada de Stern da micela. Portanto, o mecanismo de reconhecimento quiral acontece próximo à superfície da micela, na sua interface. $\mathrm{Na}$ tentativa de melhorar a discriminação quiral entre os enantiômeros, sistemas compostos por micelas e ciclodextrinas podem ser empregados. Esses sistemas são chamados de dual system ou "sistemas mistos". Esses sistemas serão discutidos posteriormente neste artigo. A Tabela 3 ilustra algumas aplicações que envolvem surfactantes quirais como seletor quiral.

\subsection{Seletores quirais baseados em antibiótico macrocíclicos}

Entre os numerosos tipos de seletores quirais empregados em eletroforese capilar, os antibióticos macrocíclicos (MA) apresentam um alto grau de enantiosseletividade para uma variada classe de enantiômeros. Esses seletores quirais

\begin{tabular}{|c|c|c|c|c|}
\hline Analito & Seletor quiral & $\begin{array}{l}\text { pH da solução } \\
\text { tampão }\end{array}$ & Matriz & Ref \\
\hline Pseudoefedrina & Poly-_-SUCLS & 2,0 & Urina & 42 \\
\hline Arotinolol & STC & 2,5 & Soro & 43 \\
\hline Warfarina & Poly-_-LSULV & 6,0 & Plasma & 44 \\
\hline Derivado benzoporfirínico mono e diácido & Colato sódico & 9,2 & Soro, microssomas & 45 \\
\hline Diltiazem e análogos & STC & 7,0 & Solução padrão & 46 \\
\hline 20 compostos farmacêuticos básicos & DDCV & - & Solução padrão & 47 \\
\hline
\end{tabular}
foram introduzidos por Armstrong e colaboradores para serem, primeiramente, empregados em cromatografia líquida de alta eficiência e, posteriormente, em eletroforese capilar ${ }^{[48]}$. Diversos seletores quirais baseados em MAs

Tabela 3 Alguns exemplos de separações quirais empregando surfactantes quirais. 

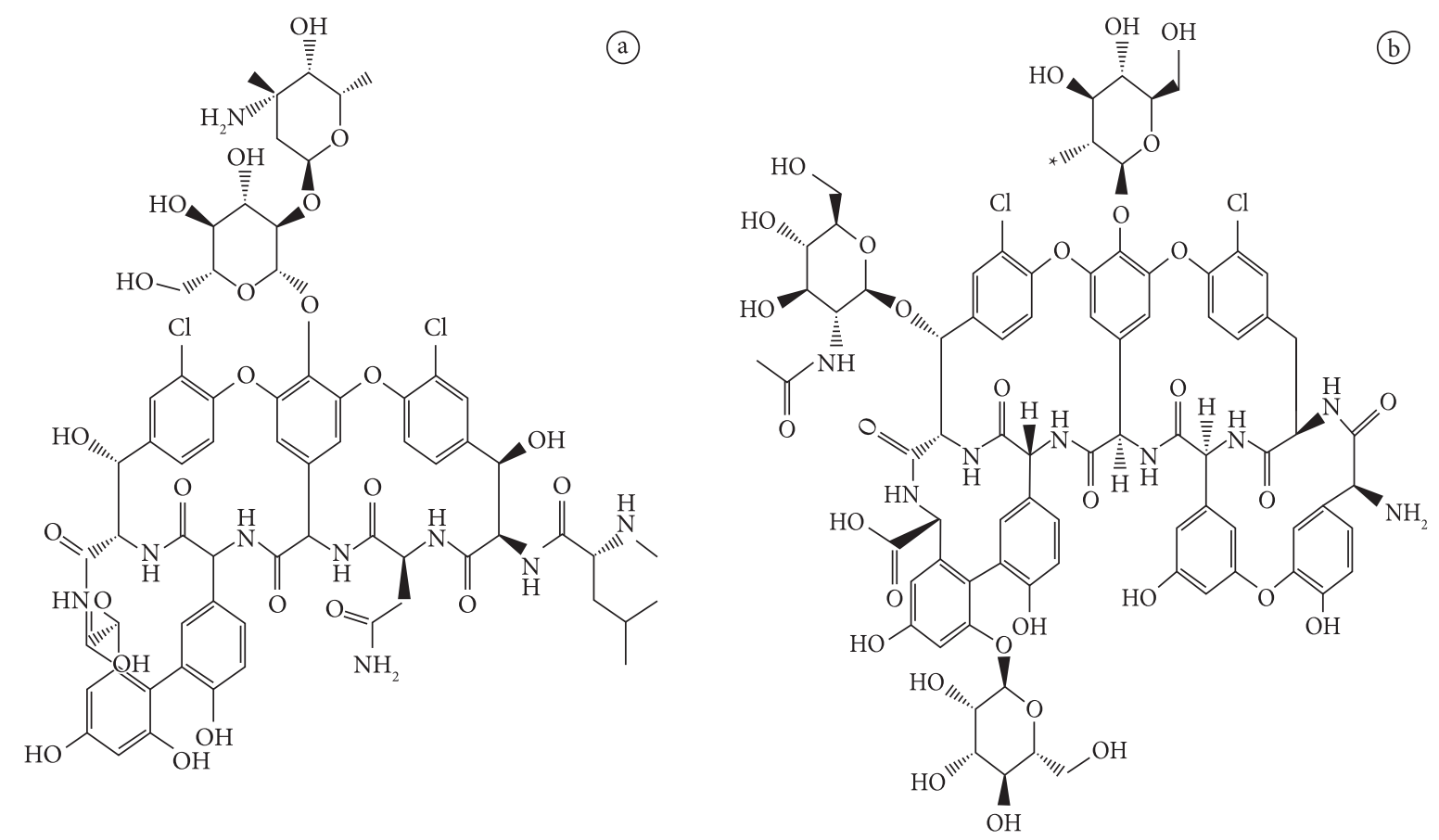

Figura 5 Estrutura química do seletor quiral vancomicina (a) e teicoplanina (b).

podem ser encontrados tais como: rifamicina $B$, rifamicina $\mathrm{SV}$, vancomicina, ristocetina $\mathrm{A}$, teicoplanina, fradiomicina, kanamicina, estreptomicina, balhimicina, actaplanina e avoparcina ${ }^{[49,50]}$. Dessa lista, merecem destaque a vancomicina (Figura 5a) e a teicoplanina (Figura 5b).

Os MAs são excelentes seletores quirais, pois apresentam inúmeros centros assimétricos que podem variar desde 9 até 38. Além disso, possuem anéis aromáticos, moléculas de açúcares, assim como grupamentos carboxílicos que estão envolvidos em interações não estereoespecíficas e estereoespecíficas. Entre essas interações, podemos citar ligações de hidrogênio, interação $\pi-\pi$, hidrofóbicas ou interações repulsivas. Além disso, a cavidade aglicona, que é uma característica de diversos MAs, permite também a formação de complexos de inclusão, favorecendo ainda mais a discriminação quiral. Grupamentos ionizáveis, que podem ser catiônicos ou aniônicos, dependendo do $\mathrm{pH}$ do meio, favorecem interações eletrostáticas entre o analito e o MAs.
Finalmente, a habilidade de enantiosseparação dos MAs foi relacionada também com a sua capacidade de sofrer dimerização ${ }^{[49]}$.

Os MAs teicoplanina e vancomicina vêm se mostrando eficientes na separação de compostos aniônicos ou compostos ácidos ionizáveis em $\mathrm{pH}$ entre 4-7. Nesse intervalo de $\mathrm{pH}$, esses seletores estão positivamente carregados, favorecendo assim a separação de compostos aniônicos, através de interações eletrostáticas. A separação de diversos enantiômeros com essas características, incluindo defensivos agrícolas, fármacos e hidroxiálcoois podem ser encontrados na literatura ${ }^{[51]}$. Portanto, trabalhos mostram que a presença de grupamentos ácidos carboxílicos nas moléculas é importante na discriminação quiral ${ }^{[52]}$.

O grande problema enfrentado com o uso de MAs como seletores quirais é sua alta absorção no UV, devido, principalmente, aos diversos anéis aromáticos presentes nessas moléculas. Essa alta absorção dificulta a detecção dos analitos, sendo, portanto, recomendável o uso de con- 
centrações muito baixas desses seletores, entre 1-5 mmol. $\mathrm{L}^{-1}$. Na tentativa de resolver esse problema, pesquisadores vêm utilizando a técnica conhecida como partial-filling countercurrent ${ }^{[49]}$. Nessa técnica, o capilar é primeiramente lavado com a solução tampão de análise (STA) sem o seletor quiral. Posteriormente, a STA contendo o seletor quiral é injetada no capilar. Antes da STA contendo o seletor quiral atingir o detector, a análise é parada. Posteriormente, a amostra é injetada e uma tensão apropriada é aplicada para que o processo eletroforético inicie a separação. Essa técnica será eficiente se os analitos e o seletor

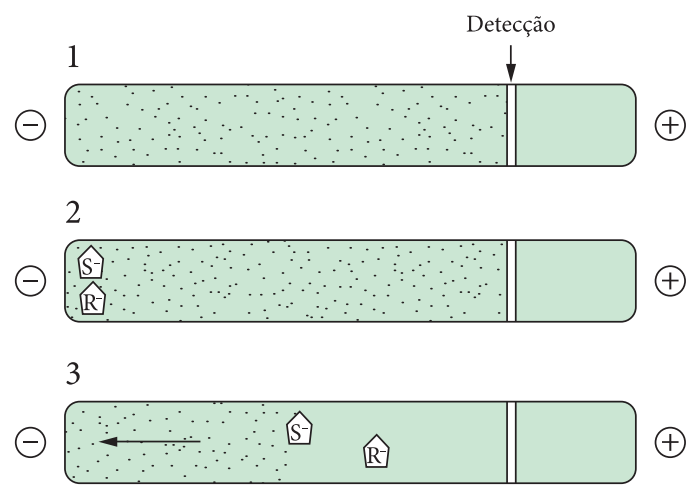

Figura 6 Esquema do processo de partial-filling countercurrent. 1) Preenchimento com a solução tampão de análise e o seletor quiral até a janela de detecção; 2) Injeção dos enantiômeros com características ácidas; 3) Aplicação de tensão e posterior análise. A seta (em 3) indica o sentido do seletor quiral após a aplicação de uma tensão. quiral migrarem em direções opostas. Portanto, é necessário que eles tenham cargas diferentes. Como exemplos, pode-se realizar a análise de um composto ácido em $\mathrm{pH}$ 6, empregando a vancomicina como seletor quiral e polaridade reversa. Nesse $\mathrm{pH}$, a vancomicina estará positivamente carregada e o analito negativamente carregado (considerando seu pKa menor que 6). Após a aplicação de uma diferença de potencial, o seletor migra em direção oposta ao detector, enquanto o analito migra em direção ao detector. Dessa forma, o seletor quiral deixa de interferir na detecção dos analitos, e os analitos, enquanto migram em direção ao detector, interagem com o MAs, podendo assim haver a separação dos enantiômeros nesse percurso. A Figura 6 ilustra esse processo de partial-filling countercurrent. A Tabela 4 apresenta algumas aplicações na análise de fármacos e metabólitos empregando os AMs como seletores quirais.

\subsection{Seletores quirais baseados em éteres de coroa}

Éteres de coroa formam complexos de inclusão estereosseletivos principalmente com aminas primárias. Além da inclusão, interações vicinais, tais como iônica, dipolo-dipolo e ligações de hidrogênio podem acontecer entre o éter de coroa e grupamentos polares do analito ${ }^{[39,60]}$.

Tabela 4 Alguns exemplos de separações quirais empregando antibióticos macrocíclicos como seletores quirais.

\begin{tabular}{lcccc}
\hline \multicolumn{1}{c}{ Analito } & Seletor quiral & $\begin{array}{c}\text { pH da solução } \\
\text { tampão }\end{array}$ & Matriz & Ref. \\
\hline AlNEs & Vancomicina & 6,0 & Solução padrão & 53 \\
Warfarina & Vancomicina & 7,0 & Solução padrão & 54 \\
Ofloxacina & Vancomicina & 4,0 & Solução padrão & 55 \\
Ácido glutâmico e aspártico & Teicoplanina & 6,0 & Solução padrão & 56 \\
Glutetimida, hexobarbital & Rifamicina SV & 7,0 & Solução padrão & 57 \\
Fármacos básicos & Rifamicina B & 7,0 & Solução padrão & 57 \\
Fenoprofeno, Cetoprofeno & Teicoplanina & 5,0 & Solução padrão & 58 \\
Loxiglumida & Vancomicina & 6,0 & Formulação farmacêutica & 59 \\
\hline
\end{tabular}


Entre os éteres de coroa disponíveis, o 6-ácido tetracarboxílico-18-coroa vem sendo amplamente empregado na separação quiral de aminoácidos, dipeptídeos e vários fármacos contendo grupamentos amina primária. A Figura 7 mostra os enantiômeros do 6-ácido tetracarboxílico-18-coroa.

\subsection{Seletores quirais baseados em proteínas}

Outros seletores quirais que podem ser empregados em análise enantiosseletiva por eletroforese capilar são as proteínas. Esses seletores podem interagir estereosseletivamente com os analitos de diversas formas, sendo que a estereosseletividade é fortemente influenciada pela estrutura terciária das proteínas ${ }^{[61]}$. A grande vantagem desses seletores quirais é que eles podem separar compostos ácidos, neutros ou básicos, dependendo de seu grau de ionização e, portanto, do $\mathrm{pH}$ da solução tampão de análise e do ponto isoelétrico da proteína. Diversas proteínas podem ser utilizadas, tais como: albumina sérica bovina, a-1-glicoproteína ácida, ovomucoide, transferrina, pepsina, avidina, caseína, penicilina $G$ acilase e outras ${ }^{[62,63]}$. O mecanismo exato de reconhecimento quiral depende do seletor em questão, não havendo um mecanismo geral aceito que possa ser usado para todas as proteínas empregadas como seletores quirais. A complexidade dessas moléculas, que possuem massa molar desde 12.000 (Citocromo C) até 150.000 (Imunoglobulina G), torna a elucidação dos mecanismos de discriminação quiral extremamente complexa. Contudo, estudos mostram que as interações hidrofóbicas, interações iônicas e ligações de hidrogênio têm um papel importante na enantiosseletividade.

O uso de proteínas em análise enantiosseletiva por CE não é muito expressivo e seu emprego como seletor quiral vem diminuindo. A

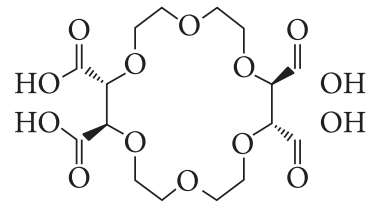

(+)-6-ácido tetracarboxílico18-coroa

Figura 7 Enantiômeros do seletor quiral 6-ácido tetracarboxílico-18-coroa.

baixa eficiência apresentada na separação (frente a outros seletores quirais), adsorção das proteínas na parede do capilar de sílica, a alta complexidade das proteínas e alta absorção na região do UV tornam esses seletores quirais não atrativos ou de segunda escolha para uso em análise enantiosseletiva por CE.

\subsection{Sistemas mistos ou dual system}

Os sistemas conhecidos como dual system ou sistemas mistos utilizam dois ou mais seletores adicionados à solução tampão de análise. Esses seletores podem ser uma mistura de seletores aquirais/quirais ou uma mistura de seletores quirais. Os seletores podem ser da mesma classe, como por exemplo, uma CD neutra e outra carregada, ou podem ser de classes diferentes, como por exemplo, uma CD e um surfactante quiral. Os sistemas mais utilizados empregam duas ciclodextrinas. Essa combinação pode levar a uma melhor resolução devido às diferenças nos mecanismos de complexação das duas CDs com os enantiômeros em relação à estabilidade de complexação, o padrão de reconhecimento quiral e a mobilidade do analito ${ }^{[64]}$. Em separações contendo duas CDs, ambos os seletores podem atuar de um modo cooperativo assim como de uma forma contrária. Crommen e colaboradores desenvolveram modelos matemáticos para explicar a enantiosseparação quando duas CDs são utilizadas ${ }^{[65]}$. Esse modelo assume que os dois seletores formam somente complexos 1:1 com os 
enantiômeros do analito e que eles levam a uma complexação independente. Contudo, os dois seletores devem ser bem caracterizados e serem isômeros isolados, o que na prática é raramente possível. Esse modelo geral é aplicável à sepa-

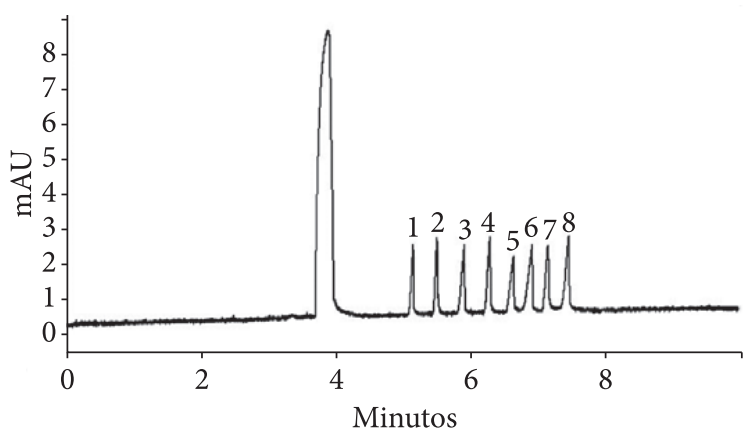

Figura 8 Eletroferograma referente à análise dos enantiômeros da hidroxicloroquina e seus metabólitos. Tampão de análise: tampão tris(hidroximetil) aminometano $100 \mathrm{mmol} . \mathrm{L}^{-1} \mathrm{pH} 9,0$ + 1\% de -CDsulfatada sódica e $30 \mathrm{mg} \cdot \mathrm{mL}^{-1}$ de HP- $\beta$-CD. Capilar de sílica fundida não recoberto de $50 \mu \mathrm{m}$ de diâmetro interno, $50 \mathrm{~cm}$ de comprimento total e $42 \mathrm{~cm}$ de comprimento efetivo, temperatura de análise de $20^{\circ} \mathrm{C}$, tensão aplicada de +18 kV. 1-(-)-(R)-HCQ, 2-(+)-(S)$H C Q$ 3-(-)-(R)-DHCQ， 4-(+)-(S)-DHCQ， 5-(-)-(R)$D C Q$, 6-(+)-(S)-DCQ e 7-(+)-(S)-BDCQ, detecção em $343 \mathrm{~nm}$. Modificado da referência $69 \mathrm{com}$ permissão da Wiley-VCH Verlag GmbH \& Co. KGaA. ração de enantiômeros neutros ou ionizados, empregando seletores quirais neutros ou carregados.

O uso desses sistemas vem crescendo e conferindo à eletroforese capilar um poder de resolução de enantiômeros ainda maior. $\mathrm{O}$ uso de CDs com surfactantes quirais ${ }^{[66]}$, CDs com seletores quirais de troca de ligantes ${ }^{[67]}$, CDs com antibióticos macrocíclicos ${ }^{[68]}$ e CDs com éteres de coroa $^{[60]}$ também tem sido descritos na literatura. A Figura 8 mostra um eletroferograma da separação quiral da hidroxicloroquina e seus metabólitos, empregando uma mistura de ciclodextrina- $\beta$-sulfatada e $2-\mathrm{HP}-\beta-\mathrm{CD}^{[69]}$. A Tabela 5 apresenta algumas separações envolvendo sistemas mistos de seletores quirais.

\section{Considerações finais}

O emprego da eletroforese capilar na análise enantiosseletiva de fármacos e metabólitos em diversas matrizes vem crescendo nas últimas décadas. A grande diversidade de seletores quirais torna possível a separação de compostos

Tabela 5 Alguns exemplos de separações quirais empregando mistura de seletores quirais.

\begin{tabular}{|c|c|c|c|c|}
\hline Analito & Seletor quiral & $\begin{array}{l}\text { pH da solução } \\
\text { tampão }\end{array}$ & Matriz & Ref. \\
\hline Tramadol & $C M-\beta-C D+M-\beta-C D$ & 10,0 & Urina & 70 \\
\hline Carvedilol & succinil- $\beta-C D+M-\beta-C D$ & 3,0 & Plasma & 71 \\
\hline Tioridazina-5-sulfóxido & $H P-\beta-C D+S-\beta-C D$ & 3,0 & Plasma & 72 \\
\hline Efaroxan & $D M-\beta-C D+C M-\beta-C D$ & 3,0 & Solução padrão & 73 \\
\hline 9-hidroxirisperidona & $\mathrm{HP}-\beta-\mathrm{CD}+\mathrm{S}-\alpha-\mathrm{CD}$ & 2,5 & Solução padrão & 74 \\
\hline Citalopram, citadiol & $S-\beta-C D+S-\beta-C D$ & 2,5 & Formulação farmacêutica & 75 \\
\hline $\begin{array}{c}\text { Propiconazol, fenbuconazol, } \\
\text { tebuconazol }\end{array}$ & $\mathrm{HP}-\gamma-\mathrm{CD}+\mathrm{SDS}$ & 3,0 & Amostras de uvas & 76 \\
\hline Ácido glicirretínico e derivados & $\beta-C D+S C$ & 8,5 & Raiz de alcaçuz & 77 \\
\hline Isoxantohumol & $\mathrm{HP}-\gamma-\mathrm{CD}+\mathrm{SDS}$ & 7,0 & Amostras de cervejas & 78 \\
\hline
\end{tabular}

S- $\beta$-CD, ciclodextrina-beta-sulfatada; $H \mathrm{P}-\gamma-C D$, hidroxipropil-gama-ciclodextrina; $C M-\beta-C D$, carboximetil-beta-ciclodextrina; CE- $\beta$-CD, carboxietil-beta-ciclodextrina; $D B-\beta-C D$, dimetil-beta-ciclodextrina; $\beta-C D$, beta-ciclodextrina; $H P-\beta-C D$, hidroxipropil-beta-ciclodextrina, $S-\alpha-C D$, ciclodextrina-alfa-sulfatada; SDS, dodecilsulfato de sódio, SC, colato de sódio; $M-\beta-C D$, metil-beta-ciclodextrina. 
extremamente complexos com diferentes características físico-químicas, aumentando muito a seletividade da técnica. Além disso, o emprego de sistemas mistos aumenta ainda mais a possibilidade de resolução de enantiômeros, que, em outras técnicas, seria praticamente impossível. Outros seletores como os líquidos iônicos quirais e a modalidade cromatografia eletrocinética quiral em microemulsão vem crescendo, aumentando ainda mais a diversidade de compostos que podem ser analisados. A grande limitação em análise enantiosseletiva por eletroforese capilar reside no alto preço de alguns seletores quirais, que aumenta o custo da análise.

\section{Referências}

1 Andersson T. Single-isomer drugs - True therapeutic advances. Clinical Pharmacokinetics 2004; 43:279. PMid:15080761. http://dx.doi.org/10.2165/00003088200443050-00001

2 Waldeck B. Biological significance of the enantiomeric purity of drugs. Chirality 1993; 5:350. http://dx.doi. org/10.1002/chir.530050514

3 Williams RC, Riley CM, Sigvardson KW, Fortunak J, Ma P, Nicolas EC et al. Pharmaceutical development and specification of stereoisomers. Journal of Pharmaceutical and Biomedical Analysis 1998;17:917. http://dx.doi.org/10.1016/S0731-7085(98)00059-4

4 Tucker GT. Chiral switches. Lancet 2000; 355:1085. http://dx.doi.org/10.1016/S0140-6736(00)02047-X

5 Branch S. Chiral Separation Techniques: A Practical Approach. 2th ed. Wiley-VCH; 2001.

6 Agranat I, Caner H, Caldwell J. Putting chirality to work: The strategy of chiral switches Nature Reviews Drug Discovery 2002; 1:753. http://dx.doi.org/10.1038/ nrd915

7 Santana FJM, Magalhães IRS, Da Fonseca P, Barth T, De Oliveira ARM. Analytical tools for the enantioselective analysis of drugs and metabolites. In: Lopes NP, Guaratini T, edtors. Analytical Chemistry for Pharmaceutical and Medical Sciences. Transworld Research Network; 2009. cap. 2, p. 19-49.
8 Scriba GKE. Cyclodextrins in capillary electrophoresis enantioseparations - Recent developments and applications. Journal of Separation Science 2008; 1931:1991.

9 Chankvetadze B. Enantioseparations by using capillary electrophoretic techniques The story of 20 and a few more years. Journal of Chromatography A 2007; 1168:45. http://dx.doi.org/10.1016/j. chroma.2007.08.008

10 Wren SAC, Rowe RC. Theoretical aspects of chiral separation in capillary electrophoresis. 1. Initial evaluation of a model. Journal of Chromatography 1992; 603:269. http://dx.doi.org/10.1016/00219673(92)85366-2

11 Vespalec RY. Principles of chiral separations by free-solution electrophoresis. In: Van Eeckhaut A, Michotte Y, edtors. Chiral Separations by Capillary Electrophoresis. CRC Press; 2010. cap. 2, p. 23-46.

12 Fanali S. Enantioselective determination by capillary electrophoresis with cyclodextrins as chiral selectors. Journal of Chromatography A 2000; 875:89. http:// dx.doi.org/10.1016/S0021-9673(99)01309-6

13 Blanco M, Valverde I. Choice of chiral selector for enantioseparation by capillary electrophoresis. TRACTrends in Analytical Chemistry 2003; 22:428. http:// dx.doi.org/10.1016/S0165-9936(03)00705-2

14 Easton CJ, Lincoln SF. Chiral discrimination by modified cyclodextrins. Chemical Society Reviews 1996; 25:163. http://dx.doi.org/10.1039/cs9962500163

15 Mikus P, Marakova K, Marak J, Nemec I, Valaskova I, Havranek E. Enantioselective analysis of pheniramine in urine by charged CD-mediated CZE provided with a fiber-based DAD and an on-line sample pretreatment by capillary ITP. Electrophoresis 2007; 28:2738. PMid:17600843. http://dx.doi.org/10.1002/ elps. 200600748

16 Glówka FK, Karazniewicz M. High performance capillary electrophoresis for determination of the enantiomers of 2-arylpropionic acid derivatives in human serum - Pharmacokinetic studies of ketoprofen enantiomers following administration of standard and sustained release tablets. Journal of Pharmaceutical and Biomedical Analysis 2004; 35:807. PMid:15193725. http://dx.doi.org/10.1016/j.jpba.2004.01.030

17 Gotti R,El-Hady DA, Andrisano V, BertucciC, El-Maali NA, Cavrini V. Determination of the chiral and achiral related substances of methotrexate by cyclodextrinmodified micellar electrokinetic chromatography. 
Electrophoresis 2004; 25:2830. PMid:15352016. http:// dx.doi.org/10.1002/elps.200405926

18 De Pablos RR, Garcia-Ruiz C, Crego AL, Marina ML. Separation of etodolac enantiomers by capillary electrophoresis. Validation and application of the chiral method to the analysis of commercial formulations. Electrophoresis 2005; 26:1106. PMid:15765483. http:// dx.doi.org/10.1002/elps.200410097

19 Chiang MT, Chang SY, Wang CW. Chiral analysis of baclofen by alpha-cyclodextrin-modified capillary electrophoresis and laser-induced fluorescence detection. Electrophoresis 2001; 22:3281. http://dx.doi. org/10.1002/1522-2683(200101)22:1\%3C123::AIDELPS123\%3E3.0.CO;2-4

20 Toussaint B, Palmer M, Chiap P. On-line coupling of partial filling-capillary zone electrophoresis with mass spectrometry for the separation of clenbuterol enantiomers. Electrophoresis 2001; 22:1363. http://dx.doi. org/10.1002/1522-2683(200105)22:7\%3C1363::AIDELPS1363\%3E3.0.CO;2-Q

21 Gübitz G, Schmid MG. Recent progress in chiral separation principles in capillary electrophoresis. Electrophoresis 2000; 21:4112. http://dx.doi. org/10.1002/1522-2683(200012)21:18\%3C4112::AIDELPS4112\%3E3.0.CO;2-T

22 De Boer T, De Zeeuw RA, De Jong GJ, Ensing $\mathrm{K}$. Recent innovations in the use of charged cyclodextrins in capillary electrophoresis for chiral separations in pharmaceutical analysis. Electrophoresis 2000; 21:3220. http://dx.doi.org/10.1002/1522$2683(20000901) 21: 15 \% 3$ C 3220 : : A D ELPS3220\%3E3.0.CO;2-X

23 Juvancz Z, Jicsinszky L, Markides KE. Phosphated cyclodextrins as new acidic chiral additives for capillary electrophoresis. Journal of Microcolumn Separation 1997; 9:581. http://dx.doi.org/10.1002/(SICI)1520667X(1997)9:8\%3C581::AID-MCS1\%3E3.0.CO;2-Y

24 Cucinotta V, Contino A, Giuffrida A, Maccarrone G, Messina M. Application of charged single isomer derivatives of cyclodextrins in capillary electrophoresis for chiral analysis. Journal of Chormatography $A$ 2010; 1217:953. PMid:20022327. http://dx.doi. org/10.1016/j.chroma.2009.11.094

25 Musenga A, Mandrioli R, Morganti E, Fanali S, Raggi MA. Enantioselective analysis of amisulpride in pharmaceutical formulations by means of capillary electrophoresis. Journal of Pharmaceutical and
Biomedical Analysis 2008; 46:966. PMid:17606354. http://dx.doi.org/10.1016/j.jpba.2007.05.022

26 Jabor VAP, Lanchote VL, Bonato PS. Enantioselective analysis of ibuprofen in human plasma by anionic cyclodextrin-modified electrokinetic chromatography. Electrophoresis 2002; 23:3041. http://dx.doi. org/10.1002/1522-2683(200209)23:17\%3C3041::AIDELPS3041\%3E3.0.CO;2-Q

27 Ywata YT, Inoue $\mathrm{H}$, Kuwayama K, Kanamori T, Tsujikawa K, Miyaguchi K et al. Forensic application of chiral separation of amphetamine-type stimulants to impurity analysis of seized methamphetamine by capillary electrophoresis. Forensic Science International 2006; 161:92. PMid:16870377. http:// dx.doi.org/10.1016/j.forsciint.2006.01.018

28 Mandrioli R, Pucci V, Sabbioni C, Bartoletti C, Fanali S, Raggi MA. Enantioselective determination of the novel antidepressant mirtazapine and its active demethylated metabolite in human plasma by means of capillary electrophoresis. Journal of Chromatography A 2004; 1051:253. PMid:15532581. http://dx.doi.org/10.1016/j.chroma.2004.05.020

29 De Santana FJM, Lanchote VL, Bonato PS. Capillary electrophoretic chiral determination of mirtazapine and its main metabolites in human urine after enzymatic hydrolysis. Electrophoresis 2008; 29:3924. PMid:18850661. http://dx.doi.org/10.1002/elps.200800053

30 Horstkötter C, Blaschke G. Stereoselective determination of ofloxacin and its metabolites in human urine by capillary electrophoresis using laser-induced fluorescence detection. Journal of Chromatography $B$ 2001; 754:169. http://dx.doi. org/10.1016/S0378-4347(00)00595-8

31 Marak J, Mikus P, Marakova K, Kaniansky D, Valaskova I, Havranek E. Enantioselective analysis of pheniramine in urine by charged CD-mediated CZE provided with a fiber-based DAD and an on-line sample pretreatment by capillary ITP. Electrophoresis 2007; 28:2738. PMid:17600843. http://dx.doi. org/10.1002/elps.200600748

32 Chou YW, Huang WS, Ko CC, Chen SH. Enantioseparation of cetirizine by sulfated-betacyclodextrin-mediated capillary electrophoresis Journal of Separation Science 2008; 28:3542.

33 Bunke A, Jira T. Chiral capillary electrophoresis using a cationic cyclodextrin. Pharmazie 1996; 51:672. 
34 Bunke A, Jira T. Use of cationic cyclodextrin for enantioseparation by capillary electrophoresis. Journal of Chromatography A 1998; 798:275. http:// dx.doi.org/10.1016/S0021-9673(97)01197-7

35 Liu Q, Inoue T, Kirchhoff JR, Huang C, Tillekeratne LMV, Olmstead $\mathrm{K}$ et al. Chiral separation of highly negatively charged enantiomers by capillary electrophoresis. Journal of Chromatography A 2004; 1033:349. PMid:15088757. http://dx.doi. org/10.1016/j.chroma.2004.01.023

36 VanEeckhautA,MichotteY.Chiralseparationsbycapillary electrophoresis: Recent developments and applications. Electrophoresis 2006; 27:2880. PMid:16688697. http:// dx.doi.org/10.1002/elps.200500375

37 Tanaka Y, Terabe S. Enantiomer separation of acidic racemates by capillary electrophoresis using cationic and amphoteric beta-cyclodextrins as chiral selectors. Journal of Chromatography A 1997; 781:151. http:// dx.doi.org/10.1016/S0021-9673(97)00489-5

38 Terabe S, Otsuka K, Ichikawa K, Tsuchya A, Ando T. Electrokinetic separations with micellar solutions and open-tubular capillaries. Analytical Chemistry 1984; 56:111. http://dx.doi.org/10.1021/ac00265a031

39 Gübitz G, Schmid MG. Chiral separation by capillary electromigration techniques. Journal of Chromatography A 2008; 1204:140. http://dx.doi. org/10.1016/j.chroma.2008.07.071

40 Otsuka K, Terabe S. Enantiomer separation of drugs by micellar electrokinetic chromatography using chiral surfactants. Journal of Chromatography $A$ 2000; 875:163. http://dx.doi.org/10.1016/S00219673(99)01167-X

41 Dey J, Ghosh A. Chiral separations by micellar electrokinetic chromatography. In: Van Eeckhaut A, Michotte Y, edtors. Chiral Separations by Capillary Electrophoresis. CRC Press; 2010. cap. 8, p. 195-234.

Rizvi SAA, Zheng J, Apkarian RP, Dublin SN, Shamsi SA. Polymeric sulfated amino acid surfactants: A class of versatile chiral selectors for micellar electrokinetic chromatography (MEKC) and MEKC-MS. Analytical Chemistry 2007; 79:879. http://dx.doi.org/10.1021/ ac061228t

43 Hefnawy MM, Micellar electrokinetic capillary chromatography determination of $(+) \mathrm{S}$ and $(-) \mathrm{R}$ arotinolol in serum using UV detection and solid phase extraction. Chirality 2002; 14:67. PMid:11748803. http://dx.doi.org/10.1002/chir.888
44 Hou J, Zheng J, Shamsi SA. Separation and determination of warfarin enantiomers in human plasma using a novel polymeric surfactant for micellar electrokinetic chromatography-mass spectrometry. Journal of Chromatography A 2007; 1159:208. http:// dx.doi.org/10.1016/j.chroma.2007.04.037

45 Peng XJ, Sternberg E, Dolphin D. Chiral separation of benzoporphyrin derivative mono- and diacids by laser induced fluorescence-capillary electrophoresis. Electrophoresis 2002; 23:93. http://dx.doi. org/10.1002/1522-2683(200201)23:1\%3C93::AIDELPS93\%3E3.0.CO;2-H

46 Nishi H, Fukuyama T, Matsuo M, Terabe S. Chiral separation of diltiazem, trimetoquinol and related compounds by micellar electrokinetic chromatography with bile salts. Journal of Chromatography A 1990; 515:133. http://dx.doi.org/10.1016/S00219673(01)89318-3

47 Peterson AG, Ahuja ES, Foley JP. Enantiomeric separations of basic pharmaceutical drugs by micellar electrokinetic chromatography using a chiral surfactant, N-dodecoxycarbonylvaline. Journal of Chromatography B 1996; 683:15. http://dx.doi. org/10.1016/0378-4347(96)00188-0

48 Armstrong DW, Rundlett K, Reid III GL. Use of a macrocyclic antibiotic, rifamycin $B$, and indirect detection for the resolution of racemic amino alcohols by CE. Analytical Chemistry 1994; 66:1690. PMid:8030783. http://dx.doi.org/10.1021/ ac00082a015

49 Fanali S, Aturki Z, D’Orazio G, Rocco A. Macrocyclic antibiotics as chiral selectors. In: Van Eeckhaut A, Michotte Y, edtors. Chiral Separations by Capillary Electrophoresis. CRC Press; 2010. cap. 5, p. 109-137.

50 Prokhorova AF, Shapovalova EN, Shpigun OA. Chiral analysis of pharmaceuticals by capillary electrophoresis using antibiotics as chiral selectors. Journal of Pharmaceutical and Biomedical Analysis 2010; 53, 1170. PMid:20675089. http://dx.doi. org/10.1016/j.jpba.2010.07.005

51 Desiderio C, Porcaro CM, Padiglioni P, Fanali S. Enantiomeric separation of acid herbicides by capillary electrophoresis using vancomycin as chiral selector. Journal of Chromatography A 1997; 781:503. http://dx.doi.org/10.1016/S0021-9673(97)00586-4

52 Hui F, Ekborg-Ott KH, Armstrong DW. Highperformance liquid chromatography and capillary electrophoretic separation of plant growth regulators and related indole compounds using 
macrocyclic antibiotics as chiral selectors. Journal of Chromatography A 2001; 906:91. http://dx.doi. org/10.1016/S0021-9673(00)00954-7

53 Ward TJ, Dann III C, Brown AP. Separation of enantiomers using vancomycin in a countercurrent process by suppression of electroosmosis. Chirality 1996; 8:77. http://dx.doi.org/10.1002/(SICI)1520636X(1996)8:1\%3C77::AID-CHIR13\%3E3.0.CO;2-P

54 Armstrong DW, Rundlett KL. CE resolution of neutral and anionic racemates with glycopeptides antibiotics and micelles. Journal of Liquid Chromatography 1995; 18:3659. PMCid:1176066. http://dx.doi. org/10.1080/10826079508014617

55 Arai $\mathrm{T}$, Nimura N, Kinoshita T. Investigation of enantioselective separation of quinolonecarboxylic acid by capillary zone electrophoresis using vancomycin as chiral selector. Journal of Chromatography A 1996; 736:303. http://dx.doi. org/10.1016/0021-9673(95)01368-7

56 Bednar P, Aturki Z, Stransky Z, Fanali F. Chiral analysis of UV nonabsorbing compounds by capillary electrophoresis using macrocyclic antibiotics. 1. Separation of aspartic and glutamic acid enantiomers. Electrophoresis 2001; 22:2129. http://dx.doi. org/10.1002/1522-2683(20017)22:11\%3C2129::AIDELPS2129\%3E3.0.CO;2-J

57 Ward TJ, Dann III C, Blaylock A. Enantiomeric resolution using the macrocyclic antibiotics rifamycin B and rifamycin SV as chiral selectors in capillary electrophoresis. Journal of Chromatography A 1995; 715:337. http://dx.doi.org/10.1016/00219673(95)00608-P

58 Gasper MP, Bethod A, Nair UB, Armstrong DW. Comparison, modeling study of vancomycin, ristocetin $\mathrm{A}$, and teicoplanin for CE enantioseparations. Analytical Chemistry 1996; 68:2501. PMid:8694258. http://dx.doi.org/10.1021/ac960154q

59 Fanali F, Desiderio C. Use of vancomycin as chiral selector in capillary electrophoresis. Optimization and quantitation of loxiglumide enantiomers in pharmaceuticals. Journal of High Resolution Chromatography 1996; 19:322. http://dx.doi. org/10.1002/jhrc.1240190604

60 Kuhn R. Enantiomeric separation by capillary electrophoresis using crown ether as chiral selector. Electrophoresis 1999; 20:2605. http://dx.doi.org/10.1002/ (SICI)1522-2683(19990901)20:13\%3C2605::AIDELPS2605\%3E3.0.CO;2-M
61 Mikus P, Marakova K. Advanced CE for chiral analysis of drugs, metabolites, and biomarkers in biological samples.Electrophoresis2009;30:2773.PMid:19653234. http://dx.doi.org/10.1002/elps.200900173

62 Haginaka J. Enantiomer separation of drugs by capillary electrophoresis using proteins as chiral selectors. Journal of Chromatography A 2000; 875:235. http://dx.doi.org/10.1016/S0021-9673(99)01168-1

63 Haginaka J. Chiral separations using proteins and peptides as chiral selectors. In: Van Eeckhaut A, Michotte Y, edtors. Chiral Separations by Capillary Electrophoresis. CRC Press; 2010. cap. 6, p. 139-161.

64 Servais A-C, Crommen J, Fillet M. Factors influencing cyclodextrin-mediated chiral separations. In: Van Eeckhaut A, Michotte Y, edtors. Chiral Separations by Capillary Electrophoresis. CRC Press; 2010. cap. 7, p. 87-107.

65 Fillet M, Hubert P, Crommen J. Enantiomeric separations of drugs using mixtures of charged and neutral cyclodextrins. Journal of Chromatography A 2000; 875:123. http://dx.doi.org/10.1016/S00219673(00)00084-4

66 Terabe S, Miyashita Y, Shibata O, Barnhart ER, Alexander LR, Patterson DG et al. Separation of highly hydrophobic compounds by ciclodextrinsmodified micellar electrokinetic chromatography. Journal of Chromatography A 1990; 516:23. http:// dx.doi.org/10.1016/S0021-9673(01)90201-8

67 Jira T, Bunke A, Karbaum A. Use of chiral and achiral ion-pairing reagents in combination with cyclodextrins in capillary electrophoresis. Journal of Chromatography A 1998; 798:281. http://dx.doi. org/10.1016/S0021-9673(97)01168-0

68 Dai R, Nie X, Li H, Saeed MK, Deng Y, Yao, G. Investigation of beta-CD-derivatized erythromycin as chiral selector in CE. Electrophoresis 2007; 28:2566. PMid:17577196. http://dx.doi.org/10.1002/ elps.200600651

69 De Oliveira ARM, Cardoso CD, Bonato PS. Stereoselective determination of hydroxychloroquine and its metabolites in human urine by liquid-phase microextraction and CE. Electrophoresis 2007; 28:1081. PMid:17295421. http://dx.doi.org/10.1002/ elps.200600420

70 Soetebeer UB, Schierenberg MO, Schulz H, Andresen P, Blaschke G. Direct chiral assay of tramadol and detection of the phase II metabolite O-demethyl tramadol glucuronide in human urine using capillary 
electrophoresis with laser-induced native fluorescence detection. Journal of Chromatography B 2001; 765:3. http://dx.doi.org/10.1016/S0378-4347(01)00366-8

71 Benh F, Michels S, Laer S, Blaschke J. Separation of carvedilol enantiomers in very small volumes of human plasma by capillary electrophoresis with laserinduced fluorescence. Journal of Chromatography $B$ 2001; 755:111. http://dx.doi.org/10.1016/S03784347(01)00045-7

72 Masetto de Gaitani C, Martinez AS, Bonato PS. Study on thioridazine 5-sulfoxide epimerization and degradation by capillary electrophoresis. Electrophoresis 2003; 24:2723. PMid:12900889. http:// dx.doi.org/10.1002/elps.200305480

73 Lorin M, Delepee R, Morin P, Ribet JP. Quantification of very low enantiomeric impurity of efaroxan using a dual cyclodextrin system by capillary electrophoresis. Analytica Chimica Acta 2007; 592:139. PMid:17512818. http://dx.doi.org/10.1016/j.aca.2007.04.018

74 Danel C, Chaminade P, Odou P, Bartemely, Azarzar D, Bonte JP et al. Enantioselective analysis of the antipsychotic 9-hydroxyrisperidone, main metabolite of risperidone, by chiral capillary EKC using dual CDs. Electrophoresis 2007; 28:2683. PMid:17600845. http://dx.doi.org/10.1002/elps.200600837

75 Sungthong B, Jac P, Scriba GKE. Development and validation of a capillary electrophoresis method for the simultaneous determination of impurities of escitalopram including the R-enantiomer. Journal of Pharmaceutical and Biomedical Analysis 2008; 46, 959. PMid:17629435. http://dx.doi.org/10.1016/j. jpba.2007.05.029

76 Wan Ibrahim WA, Hermawan D, Sanagi MM. On-line preconcentration and chiral separation of propiconazole by cyclodextrin-modified micellar electrokinetic chromatography. Journal of Chromatography A 2007; 1170:107. PMid:17915239. http://dx.doi.org/10.1016/j.chroma.2007.09.003

77 Cai J, Li P. Chemical separation of bioactive licorice compounds using capillary electrophoresis. Journal of Liquid Chromatography and Related Technologies 2007; 30:2805. PMCid:1176066. http://dx.doi. org/10.1080/10826070701588489

78 Kodama S, Yamamoto A, Sato A, Suzuki K, Yamashita T, Kemmei, $\mathrm{T}$ et al. Enantioseparation of isoxanthohumol in beer by hydroxypropyl-gammacyclodextrin-modified micellar electrokinetic chromatography. Journal of Agricultural and Food Chemistry 2007; 55:6547. 